\title{
IDH mutant and 1p/19q co-deleted oligodendrogliomas: tumor grade stratification using diffusion-, susceptibility-, and perfusion-weighted MRI
}

\author{
Yu Lin ${ }^{1} \cdot$ Zhen Xing $^{1} \cdot$ Dejun She ${ }^{1} \cdot$ Xiefeng Yang ${ }^{1}$ ' Yingyan Zheng ${ }^{1}$ Zebin Xiao ${ }^{1}$. \\ Xingfu Wang ${ }^{2} \cdot$ Dairong Cao $^{1}$ (D)
}

Received: 26 February 2017 / Accepted: 18 April 2017 /Published online: 4 May 2017

(C) The Author(s) 2017. This article is an open access publication

\begin{abstract}
Purpose Currently, isocitrate dehydrogenase (IDH) mutation and $1 \mathrm{p} / 19 \mathrm{q}$ co-deletion are proven diagnostic biomarkers for both grade II and III oligodendrogliomas (ODs). Non-invasive diffusion-weighted imaging (DWI), susceptibility-weighted imaging (SWI), and dynamic susceptibility contrast perfusion-weighted imaging (DSC-PWI) are widely used to provide physiological information (cellularity, hemorrhage, calcifications, and angiogenesis) of neoplastic histology and tumor grade. However, it is unclear whether DWI, SWI, and DSC-PWI are able to stratify grades of IDH-mutant and $1 \mathrm{p} /$ 19q co-deleted ODs.

Methods We retrospectively reviewed the conventional MRI (cMRI), DWI, SWI, and DSC-PWI obtained on 33 patients with IDH-mutated and 1p/19q co-deleted ODs. Features of cMRI, normalized ADC (nADC), intratumoral susceptibility signals (ITSSs), normalized maxim CBV (nCBV), and normalized maximum $\mathrm{CBF}$ (nCBF) were compared between low-grade ODs (LGOs) and high-grade ODs (HGOs). Receiver operating characteristic curve and logistic regression were applied to determine diagnostic performances.

Results HGOs tended to present with prominent edema and enhancement. nADC, ITSSs, nCBV, and nCBF were significantly different between groups (all $P<0.05$ ). The combination of SWI and DSC-PWI for grading resulted in sensitivity and specificity of 100.00 and $93.33 \%$, respectively.
\end{abstract}

Dairong Cao

dairongcao@163.com

1 Department of Radiology, First Affiliated Hospital of Fujian Medical University, 20 Cha-Zhong Road, Fuzhou, Fujian 350005, People's Republic of China

2 Department of Pathology, First Affiliated Hospital of Fujian Medical University, Fuzhou, China
Conclusions IDH-mutant and 1p/19q co-deleted ODs can be stratified by grades using cMRI and advanced magnetic resonance imaging techniques including DWI, SWI, and DSCPWI. Combined ITSSs with nCBV appear to be a promising option for grading molecularly defined ODs in clinical practice.

Keywords IDH mutation $\cdot 1 \mathrm{p} / 19 \mathrm{q}$ co-deletion .

Oligodendrogliomas $\cdot$ SWI $\cdot$ DSC-PWI

\section{Introduction}

Oligodendrogliomas (ODs) are glial neoplasms originated from oligodendrocytes that primarily affect supratentorial parenchyma [1]. These entities were previously classified into grades II and III according to the 2007 World Health Organization (WHO) classification system of the central nervous system (CNS). The traditional and indispensable approach relies on the histopathologic analysis of proliferative and mitotic activity, nuclear atypia, and cellularity as significant predictors of disease progression [2,3].

Historically, co-deletion of whole chromosome arms $1 \mathrm{p}$ and $19 \mathrm{q}$, namely, $1 \mathrm{p} / 19 \mathrm{q}$ co-deletion, has proved to be a diagnostic and prognostic biomarker of ODs [4-6]. However, progression-free survival (PFS) and overall survival (OS) of ODs between grades are dramatically different after adjustment for genotypes (defined by $1 \mathrm{p} / 19 \mathrm{q}$ co-deletion) [7] . Isocitrate dehydrogenase (IDH) mutation is frequently found in WHO grade II-III oligodendrogliomas and astrocytomas $[8,9]$, and is currently implicated as a prerequisite of tumorigenesis for some types of diffuse gliomas and a precondition of $1 p / 19 q$ co-deletion $[10,11]$. According to the 2016 WHO classification system, the "integrated diagnosis" of ODs requires histological classification, WHO grade, and molecular 
information (both IDH mutation and $1 \mathrm{p} / 19 \mathrm{q}$ co-deletion) [12, 13]. An oligodendroglioma-like tumor lacking diagnostic mutations is given a "not otherwise specified (NOS)" designation, which is strongly discouraged by neuro-oncologists. When dealing with NOS ODs, care should be taken throughout to exclude CNS tumors including astrocytomas, glioblastomas, clear cell ependymoma, and dysembryoplastic neuroepithelial tumor [12]. In addition, nearly all histological "oligoastrocytomas" can be reclassified as either astrocytomas or ODs using genetic testing $[12,14]$.

Diffusion-weighted imaging (DWI) could reflect the Brownian movement of water molecules and the cytogenetic profile of cerebral tumors through the measurement of apparent diffusion coefficient (ADC) values $[15,16]$. As another advanced magnetic resonance imaging (aMRI) approach, susceptibility-weighted imaging (SWI) utilizes intratumoral susceptibility signals (ITSSs) to demonstrate tumor vascularity and blood metabolite sensitively $[17,18]$. Regional cerebral blood volume (rCBV) and regional cerebral blood flow (rCBF) derived from dynamic susceptibility contrast perfusion-weighted imaging (DSC-PWI) provide hemodynamic information of microvasculature in glioma grading and genotyping [19-24]. Non-invasive measurement of rCBV has been confirmed to correlate with tumor vascularity and clinical prognosis of gliomas, which has the potential to provide additional information to conventional MRI (cMRI) $[7,19,21]$. As recently revealed, IDH mutations or $1 \mathrm{p} / 19 \mathrm{q}$ codeletions correlate with aberrant cellularity and angiogenesis by regulating proliferation and vascularization $[6,19,21,25]$. Therefore, we suspected that the inclusion of mixed genetic profiles in previous studies might lead to a low prediction accuracy of aMRI techniques for grading ODs [7, 26].

During the past two decades, extensive studies have focused on the surrogate MRI characters of tumor grade (defined by histopathology) and genetic subtype (defined by $1 \mathrm{p} / 19 \mathrm{q}$ co-deletion or IDH mutation) of CNS tumors [19, $23,24,27,28]$, whereas these two genic alterations act as diagnostic markers rather than grouping factors for ODs [12]. Thus, it is inappropriate for the neuropathologists or neuroradiologists to "genotype" ODs following the WHO update. To our knowledge, there is no aMRI research in presurgical grading of the redefined tumors. Therefore, the present study aims to investigate whether multiparametric MRI is predictive of tumor grades of the new entity.

\section{Methods}

\section{Patients}

This retrospective study was approved by the institutional review board, and patient informed consent requirement was waived. Patients with oligodendroglial tumors were histologically confirmed through image-guided stereotactic biopsy or tumor biopsy on resection performed at our hospital from January 2014 to January 2017. The histopathologic diagnosis was made by experienced neuropathologists according to the updated WHO classification standards. The inclusion criteria were (1) a histopathology diagnosis of ODs or oligoastrocytomas according to 2007 WHO classification; (2) 3.0 Tesla cMRI scans combined with DWI, SWI, and DSCPWI before any intervention; and (3) a known IDH mutation and $1 \mathrm{p} / 19 \mathrm{q}$ co-deletion status for reclassification according to 2016 WHO guidelines.

\section{Conventional MRI}

The MR examinations were performed in the routine clinical workup using a 16-channel dedicated head matrix coil on a 3.0 Tesla MRI system (MAGNETOM Verio; Siemens Healthcare, Erlangen, Germany). The sequences of cMRI protocols were gradient-echo T1-weighted imaging (T1WI, TR/TE $=250 \mathrm{~ms} /$ $2.48 \mathrm{~ms}$ ), turbo spin-echo T2-weighted imaging (T2WI, TR/ $\mathrm{TE}=4000 \mathrm{~ms} / 96 \mathrm{~ms}$ ), fluid-attenuated inversion recovery (FLAIR, TR/TE $=9000 \mathrm{~ms} / 94 \mathrm{~ms}$, TI $2500 \mathrm{~ms}$ ) imaging, and gradient-echo contrast-enhanced T1WI (CE-T1WI, TR/ $\mathrm{TE}=250 \mathrm{~ms} / 2.48 \mathrm{~ms}$ ). All images were acquired with field of view (FOV) of $220 \mathrm{~mm} \times 220 \mathrm{~mm}$, and slice thickness of $5 \mathrm{~mm}$.

\section{Advanced MRI}

DWI was performed in the axial plane with a spin-echo-planar sequence $(\mathrm{TR} / \mathrm{TE}=8200 / 102 \mathrm{~ms}, \mathrm{NEX}=2.0)$. Corresponding ADC maps were generated automatically by the MRI system. Diffusion gradients were encoded in three orthogonal directions to accentuate the effects of diffusion.

Non-enhanced SWI was performed using a 3D fully flowcompensated gradient-echo sequence $(\mathrm{TR} / \mathrm{TE}=27 / 20 \mathrm{~ms}$, flip angle $=15^{\circ}$ ) and a reconstruction by combining the magnitude and phase images.

DSC-PWI was achieved with a gradient-recalled echo-planar sequence $\left(\mathrm{TR} / \mathrm{TE}=1000-1250 / 54 \mathrm{~ms}\right.$, flip angle $=35^{\circ}$, NEX $=1.0$ ). In the first three phases, non-enhanced images were scanned to establish a precontrast baseline. For the scan in the fourth phase of DSC-PWI, a standard dose $(0.1 \mathrm{mmol} /$ $\mathrm{kg}$ ) of gadobenate dimeglumine (Gd-BOPTA) was injected intravenously at a flow rate of $3.00 \mathrm{ml} / \mathrm{s}$ through a catheter inserted into the antecubital vein, followed by a $20-\mathrm{ml}$ continuous saline flush. A series of 60 phases and a total of 1200 images were constantly produced in $96 \mathrm{~s}$.

\section{Image analysis}

All imaging assessments were performed with the standard software integrated in the MRI system including syngo.MR 
Neuro Perfusion Engine (syngo.via; Siemens Healthcare, Erlangen, Germany). Two neuroradiologists (with 30 and 9 years of experience, respectively) were blinded to the pathological features, and the cMRI sequences were evaluated individually. The senior made the final decision when there was disagreement between them. The following characteristics were qualitatively evaluated: tumor location (assessed by primary lobe of involvement; frontal, parietal, temporal, occipital, insular, and other sites), signal intensity (homogeneous vs. heterogeneous), tumor borders (sharp/smooth vs. indistinct/irregular); peritumoral edema (presence vs. absence), and contrast enhancement pattern (no/blurry vs. nodular/ ring-like).

To assess DWI data, ADC values were measured by manually placing regions of interest (ROIs) on the ADC maps. At least five non-overlapping ROIs (size 25$40 \mathrm{~mm}^{2}$ ) were placed inside the tumor areas of visually lowest $\mathrm{ADC}$. The minimum $\mathrm{ADC}\left(\mathrm{ADC}_{\min }\right)$ was calculated as the mean value from the ROI of the lowest ADC. The ROIs' placement was made from the solid portion (defined on FLAIR images and CE-T1WI) to avoid hemorrhagic, calcified, necrotic, and cystic regions that might affect the measurements. To minimize variances in $\mathrm{ADC}_{\text {min }}$ values, normalized ADC (nADC) was derived from the ratios of the tumor $\mathrm{ADC}_{\text {min }}$ to the mean $\mathrm{ADC}$ of the contralateral normal-appearing white matter (CNWM, defined on T2WI and CE-T1WI).

Minimum intensity projection technique at 2-mm slice thickness was conducted for the semi-quantitative analysis of SWI. Intratumor calcification was identified and excluded using phase image cross-correlation analysis or corresponding CT scans. The degree of ITSSs included four grades as previously described [29, 30]: (1) grade 0 with no ITSS; (2) grade 1 with 1-5 dot-like or linear ITSSs; (3) grade 2 with 6-10 dotlike or linear ITSSs; and (4) grade 3 with more than 11 dot-like or linear ITSSs.

To evaluate DSC-PWI data, rCBV and rCBF maps were generated automatically using a single-compartment model and an arterial input function. Each value was measured using the same method in the previous nADC assessments by targeting high $\mathrm{rCBV} / \mathrm{rCBF}$. At least five non-overlapping ROIs were placed inside the tumor areas of visually highest $\mathrm{rCBV} / \mathrm{rCBF}$. The maximum CBV/CBF was calculated as the mean value from the ROI of the greatest $\mathrm{rCBV} / \mathrm{rCBF}$. The normalized maximum rCBV (nCBV) and normalized maximum $\mathrm{rCBF}$ (nCBF) were calculated by normalizing to the CNWM.

All the parameters derived from DWI and DSC-PWI were measured by a qualified neuroradiologist who was experienced in image processing and analysis, and blinded to the tumor pathology. The methods used in the analysis of DWI, SWI, and DSC-PWI have been proven to provide the highest interobserver and intraobserver reproducibility $[27,29,30]$.

\section{Molecular studies}

Immunohistochemistry (IHC) staining was performed using 5 - $\mu \mathrm{m}$-thick sections in paraffin-embedded specimens and anti-IDH1 (R132) (clone DIA-H09, Dianova Inc., Hamburg, Germany) at a dilution of 1:500. Samples were scored positive when IDH1-R132 staining showed more than $10 \%$ of the tumor cells.

IDH1/2 alterations in the hotspot codons R132 and R172 were also simultaneously assessed using bidirectional cycle sequencing of polymerase chain reaction (PCR)-amplified fragments with the standard Sanger method.

$1 p / 19 q$ deletions were detected by fluorescence in situ hybridization (FISH) analysis on 5 - $\mu \mathrm{m}$-thick paraffin-embedded sections using Vysis 1p36/1q25 and 19q13/19p13 dual-color probes according to the manufacturer's instructions (Vysis Inc., Illinois, USA). Signals were scored with at least 100 non-overlapping intact nuclei. $1 \mathrm{p} / 19 \mathrm{q}$ co-deletion was defined as the signal ratio of $1 \mathrm{p} / 1 \mathrm{q}<0.70$ and $19 \mathrm{q} / 19 \mathrm{p}<0.70$.

\section{Statistical analyses}

Quantitative data were described as mean \pm standard deviation (SD). Interobserver variabilities of cMRI features and ITSSs levels were analyzed by kappa statistics. Parameters derived from aMRI between low-grade ODs (LGOs) and high-grade ODs (HGOs) were compared using Mann-Whitney $U$ test. Either Pearson's chi-square test or Fisher's exact test was performed for categorical data. The receiver operating characteristic (ROC) curves and binary logistic regression analysis were carried out to decide diagnostic accuracy and optimum cutoff value. The sensitivity, specificity, positive predictive value (PPV), negative predictive value (NPV), Youden index (YI), and area under the curve (AUC) were calculated based on the optimal threshold for each parameter and combination. Statistical analysis was conducted using Statistical Package for the Social Sciences (SPSS), version 19.0.0 (SPSS Inc., Chicago, IL, USA), and MedCalc, version 12.1.0 (MedCalc Inc., Mariakerke, Belgium). A significant difference was defined as a $P$ value less than 0.05 .

\section{Results}

Thirty-three cases (17 males and 16 females, age $42.97 \pm 9.03$ years) were diagnosed as IDH-mutant and $1 \mathrm{p} /$ $19 \mathrm{q}$ co-deleted ODs based on the current WHO criteria. DWI, SWI, and DSC-PWI combined with cMRI were performed on all patients. Five cases of DSC-PWI were of poor perfusion quality and excluded in further analysis.

Table 1 shows the summarization of the main clinical and cMRI features of molecularly defined (IDH-mutant and $1 p / 19 q$ co-deleted) ODs between groups. All kappa coefficients of 
Table 1 The main clinical and cMRI features of LGOs and HGOs

\begin{tabular}{|c|c|c|c|c|}
\hline & LGO $(n=18)$ & $\operatorname{HGO}(n=15)$ & Total & $P$ value \\
\hline Gender (male/female) & $9 / 9$ & $8 / 7$ & $17 / 16$ & 0.849 \\
\hline Age (years) & $41.56 \pm 9.03$ & $44.67 \pm 9.05$ & $42.97 \pm 9.03$ & 0.319 \\
\hline Location & & & & 0.134 \\
\hline Frontal & 11 & 13 & 24 & \\
\hline Parietal & 2 & 1 & 3 & \\
\hline Temporal & 2 & 0 & 2 & \\
\hline Occipital & 1 & 0 & 1 & \\
\hline Insular & 2 & 0 & 2 & \\
\hline Others & 0 & 1 & 1 & \\
\hline Signal & & & & 0.134 \\
\hline Homogeneous & 7 & 2 & 9 & \\
\hline Heterogeneous & 11 & 13 & 24 & \\
\hline Border & & & & 0.722 \\
\hline Sharp/smooth & 6 & 4 & 10 & \\
\hline Indistinct/irregular & 12 & 11 & 23 & \\
\hline Edema & & & & 0.013 \\
\hline Presence & 6 & 12 & 18 & \\
\hline Absence & 12 & 3 & 15 & \\
\hline Enhancement & & & & 0.005 \\
\hline No/blurry & 14 & 4 & 18 & \\
\hline Nodular/ring-like & 4 & 11 & 15 & \\
\hline
\end{tabular}

A italicized $p$ value indicate a significant difference between results $(p<0.05)$ interobserver measurements were greater than 0.75 (an excellent agreement). Out of 33 patients, 11/18 (61.1\%) LGOs and $13 / 15(86.7 \%)$ HGOs situated in the frontal lobe with insignificant difference between grades. Heterogeneous appearance (including hemorrhage and cystic degeneration) and infiltrating borders were non-specifically observed in ODs regardless of grades. It is noteworthy that LGOs revealed a decreased level of edema compared to anaplastic counterparts $(P=0.005)$. Additionally, HGOs were related to the nodular or ring-like enhancement patterns on CE-T1WI $(P=0.002)$.

Tables 2 and 3 give the (semi-)quantitative measurements of aMRI for LGOs and HGOs. Relevant MRI images are depicted in Figs. 1 and 2. nADC values from DWI were significantly higher in LGOs than in HGOs $(P<0.001)$. ITSS levels of HGOs were substantially greater than that of LGOs with seven (46.7\%) HGOs which exerted an agglomerated or nodular appearance of ITSSs $(P=0.006)$. Both nCBV and

Table 2 Comparison of variables derived from DWI and DSC-PWI

\begin{tabular}{llll}
\hline & LGO $($ mean \pm SD $)$ & HGO $($ mean \pm SD) & $\boldsymbol{P}$ value \\
\hline nADC & $1.36 \pm 0.27$ & $0.96 \pm 0.12$ & $<0.001$ \\
nCBV & $2.34 \pm 1.16$ & $5.08 \pm 1.81$ & $<0.001$ \\
$\mathrm{nCBF}$ & $2.81 \pm 1.26$ & $5.45 \pm 2.64$ & 0.005 \\
\hline
\end{tabular}

A italicized $p$ value indicate a significant difference between results $(p<0.05)$
$\mathrm{nCBF}$ values in HGOs were significantly higher than the values of LGOs $(P<0.001$ and $P=0.005)$.

The results of ROC analysis of the parameters are illustrated in Table 4. Optimal cutoff values of quantitative nADC from DWI and quantitative nCBV from DSC-PWI in tumor grading were determined as 1.11 and 2.90 , respectively. The sensitivity/specificity of nADC and nCBV were 100.00/77.78 and $92.31 / 80.00 \%$, respectively. With a threshold value of $\geq 1$ for semi-quantitative ITSSs from SWI, the sensitivity and specificity in the diagnosis of HGOs were 60.00 and $83.33 \%$, respectively.

Logistic regression and ROC analyses were conducted for cMRI (combining edema and enhancement) and aMRI (combining DWI, SWI, and DSC-PWI by two or three parameters). The combination of SWI and DSC-PWI resulted in the highest sensitivity and specificity of 100.00 and $93.33 \%$, respectively. Nevertheless, combined DWI, SWI, and DSC-PWI techniques yielded similar diagnostic performance.

\section{Discussion}

Significant concordance between the histopathological grading and aMRI-based grading was found in our reclassified cases. This concordance may be of more clinical value as we followed the updated WHO classification standards which have already redefined many infiltrating gliomas. Our study 
Table 3 Comparison of ITSS levels derived from SWI $(P=0.006)$

\begin{tabular}{lccc}
\hline ITSS level & LGOs & HGOs & Total \\
\hline 0 & 7 & 1 & 8 \\
1 & 8 & 5 & 13 \\
2 & 1 & 3 & 4 \\
3 & 2 & 6 & 8 \\
Total & 18 & 15 & 33 \\
\hline
\end{tabular}

has revealed non-invasive MRI techniques including DWI, SWI, and DSC-PWI which may improve the diagnostic accuracy in grading redefined ODs.

Although molecular and genetic factors have been introduced recently, a large body of evidence has suggested that glioma grading based on histopathological evaluation is still important for determining therapeutic options and predicting clinical outcomes $[2,5,12,31,32]$. Shaw et al. suggest that OS (approximately 4 years of HGOs and 10 years of LGOs) and treatment planning are different between grades [31-33]. Patients with LGOs can benefit from subtotal resection and early radiotherapy with small fraction doses, or remain stable without any treatment [5, 34, 35]. In contrast, several randomized controlled trials of HGOs show that adjuvant chemotherapy increases PFS while postoperative radiotherapy produces minimum benefit $[33,36]$. Besides, preoperative MRI-based grading and guidance may affect the surgical strategy to maximize safe resection $[31,37]$.
Frontal location, characteristic calcification, poorly defined border, and internal heterogeneity are highly suggestive of molecularly defined ODs as unveiled in the latest studies [27, 38-41], which may serve as reliable and robust biomarkers in the differential diagnosis prior to tumor grading. The results of this study display that molecularly defined ODs have a predilection for the frontal lobes that is consistent with previous findings [27]. Peritumoral edemas in HGO patients are frequently encountered in our study, mainly attributed to the high frequency of cellular/vasogenic pathogenesis in high-grade tumors [42]. Up to date, Johnson et al. have suggested that the tumor borders of molecularly defined ODs are fairly infiltrative [41, 43]. While peritumoral edema was determined based on its non-enhancement on CE-T1WI and higher signal on FLAIR in contrast to clearly enhanced tumor solid component, radiologically, definite differentiation of tumor infiltration from peritumoral edema is still a technical challenge in the field. Although nodular or ring-like enhancement in association with blood-brain barrier breakdown and angiogenesis [37, 42] is frequently observed in HGOs, it should be further noted that such enhancement patterns of HGOs are more homogenous than that of glioblastoma (WHO grade IV, most are IDH wild types), whereas it is quite difficult to stratify tumor grades by utilizing cMRI only.
Fig. 1 A 55-year-old man with molecularly defined LGO. On axial T2WI (a), the lesion shows homogeneous signal. Axial CET1WI (b) demonstrates absence of contrast enhancement. Corresponding ADC maps (c) show an increased $A D C$ value $(\mathrm{nADC}=1.52)$. rCBV maps $(\mathbf{d})$ show significantly reduced perfusion with the calculated nCBV of 1.12. SWI (e) presents nearly no evidence of ITSS. H\&E staining $(\times 100)$ photomicrograph $(\mathbf{f})$ reveals a low cell density
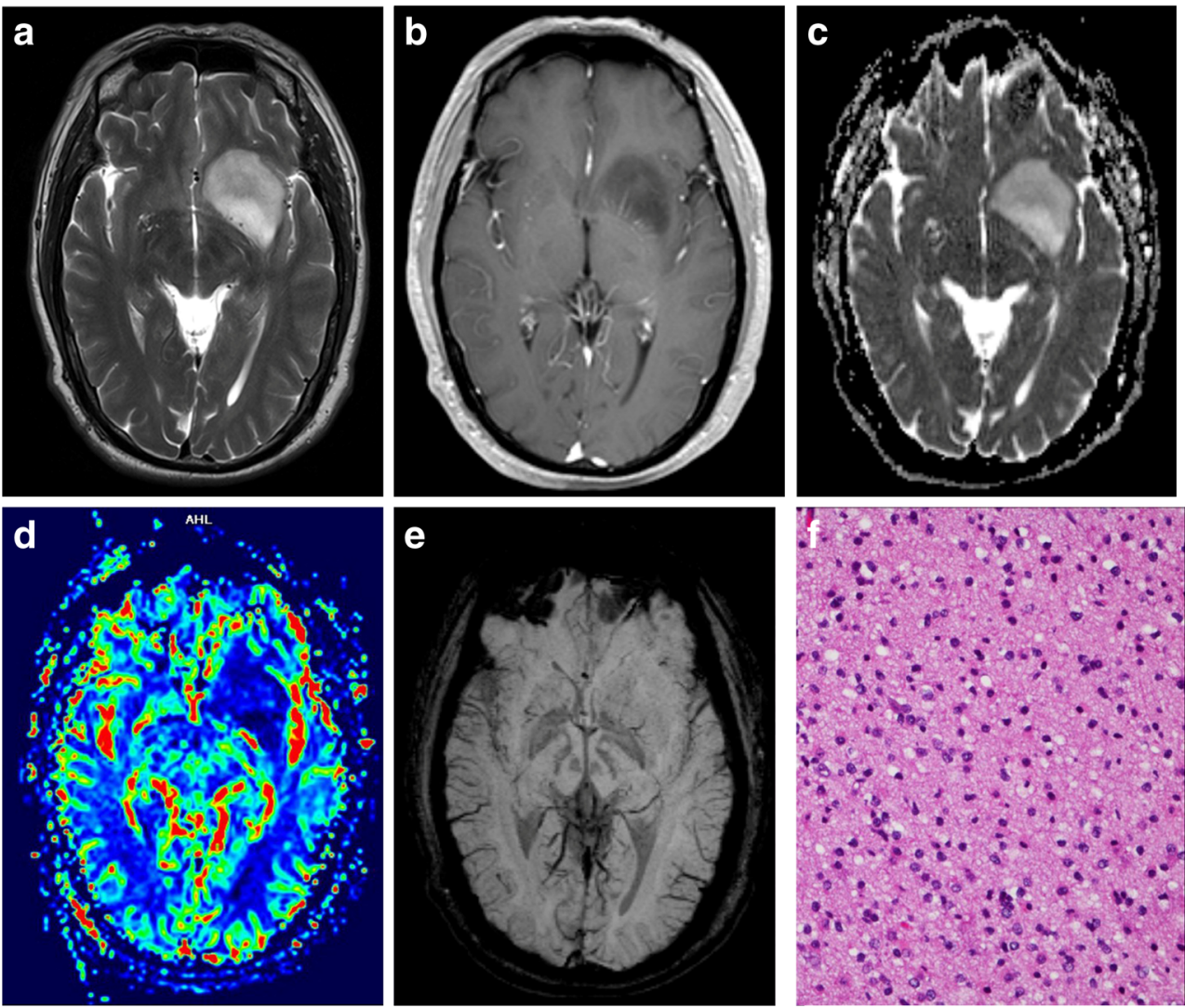

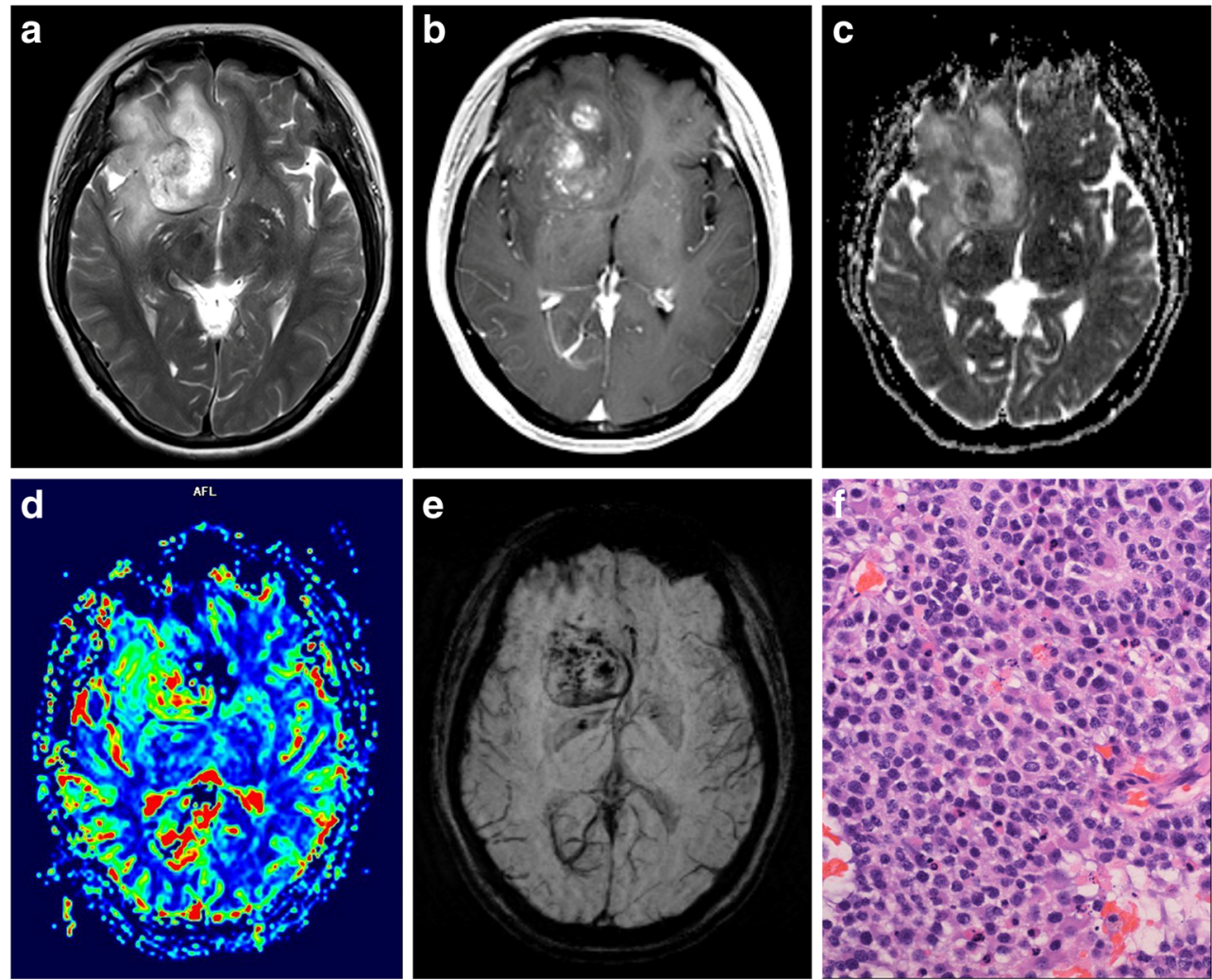

Fig. 2 A 50-year-old woman with molecularly defined HGO. On axial T2WI (a), the lesion shows heterogeneous signals with possible cystic or necrotic regions. Markedly nodular contrast enhancement is demonstrated on CE-T1WI (b). Corresponding ADC maps (c) exhibit a partially decreased $\mathrm{ADC}$ value $(\mathrm{nADC}=0.85)$. rCBV maps $(\mathbf{d})$ show significantly elevated perfusion with a nCBV value of 4.69. SWI reveals (e) a maximum degree of ITSSs. H\&E staining $(\times 400)$ photomicrograph (f) reveals relatively high cellularity and proliferative vessels
Although nADC values were not significantly different between WHO grades in morphologically defining ODs (based on WHO 2007 criteria) [26], molecularly defined ODs could be stratified by grades applying nADC as shown in our research. Diffusion MRI enables studies in tumorous physical properties microscopically: increased proliferation elevates cellularity and restricts water diffusion [15, 44]. Mutations of the IDH gene family produces oncometabolite 2hydroxyglutarate, leading to a slower growth of tumor cells compared to the wild types [9]. Conversely, a poor correlation between $1 \mathrm{p} / 19 \mathrm{q}$ co-deletion and cell proliferation was confirmed in an early study [27]. Consequently, it is reasonable to speculate that so-called IDH wild-type ODs of various proportions in both grades may play as confounding factors and result in a complexity of heterogeneity in preceding studies.

SWI appears to be a promising imaging method in grading gliomas based on the different frequencies and appearances of ITSSs [18]. The dot-like hypointense signal is regarded as
Table 4 Cutoff, sensitivity (\%), specificity (\%), PPV (\%), NPV (\%), YI, and AUC of parameters for grading OD

\begin{tabular}{lccccrrr}
\hline & Cutoff & Sensitivity & Specificity & PPV & NPV & YI & AUC \\
\hline cMRI & & 73.33 & 77.78 & 72.97 & 78.09 & 0.51 & 0.76 \\
nADC (DWI) & 1.11 & 100.00 & 77.78 & 78.64 & 100.00 & 0.78 & 0.92 \\
ITSS (SWI) & 1 & 60.00 & 83.33 & 74.65 & 71.80 & 0.43 & 0.77 \\
nCBV (DSC-PWI) & 2.90 & 92.31 & 80.00 & 79.06 & 92.71 & 0.72 & 0.90 \\
DWI + SWI & & 73.33 & 100.00 & 100.00 & 82.09 & 0.73 & 0.95 \\
DWI + DSC-PWI & & 92.31 & 93.33 & 91.89 & 93.68 & 0.86 & 0.94 \\
SWI + DSC-PWI & & 100.00 & 93.33 & 92.46 & 100.00 & 0.93 & 0.99 \\
aMRI & & 100.00 & 93.33 & 92.46 & 100.00 & 0.93 & 0.99 \\
\hline
\end{tabular}

$P P V$ positive predictive value, $N P V$ negative predictive value, $Y I$ Youden index, $A U C$ area under the curve 
microhemorrhage and the linear signal as an intralesional vessel, both of which are common in malignant CNS tumors [30]. Similarly, the results of our study indicate a significant difference in ITSSs between grades, suggesting that ITSSs can be a valuable biomarker for a precise diagnosis of HGO. However, medium-to-high levels of ITSSs in LGOs were occasionally observed, which may be attributed to the moderately increased neovascularity in both LGOs and HGOs as a consequence of $1 \mathrm{p} / 19 \mathrm{q}$ co-deletion $[21,40]$.

Our study introduced multiple diagnostic parameters derived from DSC-PWI to identify high-grade IDH-mutant and $1 p / 19 q$ co-deleted ODs. We believe that $n C B V$ and $n C B F$ values can be used as surrogates of angiogenic features of molecularly defined ODs. A significant positive correlation was found between tumor grades and $\mathrm{nCBV}$ values. However, most of the previous researchers [7, 24, $25,28,45]$ indicated that $\mathrm{nCBV}$ was either inefficient or overlapping in grading ODs when using the gold standard of 2007 WHO criteria. With improved understanding of the pathogenic mechanism, scientists suggest that IDH mutation can reduce the activation of hypoxia-inducible factor1 alpha and inhibit downstream signaling pathways of angiogenesis $[19,46]$. Similarly, some studies indicated that gliomas with $1 \mathrm{p} / 19 \mathrm{q}$ co-deletion were likely to have mildly elevated rCBV due to increased microvascular proliferation $[21,25]$. Indeterminate proportions of "genotypes" may substantially increase the overlap of perfusion parameters between LGOs and HGOs suggested in previous studies $[7,25]$.

The combination of ITSSs and nCBV remarkably improved the grading accuracy, and may serve as an optimal method neuroradiologically. However, the combination of all aMRI methods provided no improvement in differentiating anaplastic tumors from LGOs. Although nADC is an ideal parameter for grading molecularly defined ODs, additional DWI procedures may not be necessary when both SWI and DSC-PWI are available.

There are some limitations in our study such as the retrospective nature and small sample. Although the case number of IDH-mutant and $1 \mathrm{p} / 19 \mathrm{q}$ co-deleted OD is relatively limited due to its low prevalence and the complexity of multiple cytogenetic characteristics required for diagnosis, further study is needed in multicenter large-scale MRI-based radiomics approaches. Moreover, a possible contrast extravasation in DSC-PWI may generate an imprecise estimation of glioma nCBV. Both preload of gadolinium and leakage correction may be necessary to minimize the leakage contamination. In addition, intratumoral calcific, cystic, necrotic, and hemorrhagic areas may potentially interfere with our manual measurements despite the efforts made to minimize the interference. Finally, a long-term follow-up is desired for analysis of patients' prognosis.

\section{Conclusions}

The study demonstrates that IDH-mutant and $1 \mathrm{p} / 19 \mathrm{q}$ codeleted ODs can be stratified by grades using cMRI and aMRI techniques including DWI, SWI, and DSC-PWI with high specificity and sensitivity. HGOs are prone to prominent edema and enhancement. Furthermore, adding ITSSs to nCBV in clinical practice seems to be a promising option for grading molecularly defined ODs.

\section{Compliance with ethical standards}

Funding This study was funded by the Leading Project of the Department of Science and Technology of Fujian Province (No. 2016Y0101), the Special Funds of Provincial Finance of Fujian Province (No. BPB-CDR2013) and the Research Foundation for Young Scholars of the Health Department of Fujian Province (No. 2013-1-34).

Conflict of interest The authors declare that they have no conflict of interest.

Ethical approval All procedures performed in the studies involving human participants were in accordance with the ethical standards of the institutional and/or local ethical committee and with the 1964 Helsinki Declaration and its later amendments or comparable ethical standards.

Informed consent For this type of study formal consent is not required.

Open Access This article is distributed under the terms of the Creative Commons Attribution 4.0 International License (http:// creativecommons.org/licenses/by/4.0/), which permits unrestricted use, distribution, and reproduction in any medium, provided you give appropriate credit to the original author(s) and the source, provide a link to the Creative Commons license, and indicate if changes were made.

\section{References}

1. Fortin D, Cairncross GJ, Hammond RR (2000) Oligodendroglioma: an appraisal of recent data pertaining to diagnosis and treatment. Neurosurgery 45:1279-1991

2. Fuller GN, Scheithauer BW (2007) The 2007 revised World Health Organization (WHO) classification of tumours of the central nervous system: newly codified entities. Brain Pathol 17:304-307

3. Giannini C, Scheithauer BW, Weaver AL et al (2001) Oligodendrogliomas: reproducibility and prognostic value of histologic diagnosis and grading. J Neuropathol Exp Neurol 60:248262

4. Ducray F, Idbaih A, Reyniès AD et al (2008) Anaplastic oligodendrogliomas with $1 \mathrm{p} 19 \mathrm{q}$ codeletion have a proneural gene expression profile. Mol Cancer 7:1-17

5. Zhang ZY, Chan AK, Ng HK et al (2014) Surgically treated incidentally discovered low-grade gliomas are mostly IDH mutated and 1p19q co-deleted with favorable prognosis. Int J Clin Exp Patho 7: $8627-8636$

6. Frenel JS, Leux C, Loussouarn D et al (2013) Combining two biomarkers, IDH1/2 mutations and $1 \mathrm{p} / 19 \mathrm{q}$ codeletion, to stratify anaplastic oligodendroglioma in three groups: a single-center experience. J Neuro-Oncol 114:85-91 
7. Jenkinson MD, Smith TS, Joyce KA et al (2006) Cerebral blood volume, genotype and chemosensitivity in oligodendroglial tumours. Neuroradiology 48:703-713

8. Hartmann C, Meyer J, Balss J et al (2009) Type and frequency of IDH1 and IDH2 mutations are related to astrocytic and oligodendroglial differentiation and age: a study of 1,010 diffuse gliomas. Acta Neuropathol 118:469-474

9. Jalbert LE, Elkhaled A, Phillips JJ et al (2017) Metabolic profiling of IDH mutation and malignant progression in infiltrating glioma. Scientific reports 7:44792

10. Watanabe T, Nobusawa S, Kleihues P et al (2009) IDH1 mutations are early events in the development of astrocytomas and oligodendrogliomas. Am J Pathol 174:1149-1153

11. Mcalisterhenn L (2009) IDH1 and IDH2 mutations in gliomas. New Engl J Med 360:765-773

12. Louis DN, Perry A, Reifenberger G et al (2016) The 2016 World Health Organization classification of tumors of the central nervous system: a summary. Acta Neuropathol 131:803-820

13. Olar A, Sulman EP (2015) Molecular markers in low grade glioma- toward tumor reclassification. Semin Radiat Oncol 25:155163

14. Sahm F, Reuss D, Koelsche C et al (2014) Farewell to oligoastrocytoma: in situ molecular genetics favor classification as either oligodendroglioma or astrocytoma. Acta Neuropathol 128:551-559

15. Kang Y, Choi SH, Kim YJ et al (2011) Gliomas: histogram analysis of apparent diffusion coefficient maps with standard- or high-bvalue diffusion-weighted MR imaging - correlation with tumor grade. Radiology 261:882-890

16. Yan R, Haopeng P, Xiaoyuan F et al (2016) Non-Gaussian diffusion MR imaging of glioma: comparisons of multiple diffusion parameters and correlation with histologic grade and MIB-1 (Ki-67 labeling) index. Neuroradiology 58:121-132

17. Sehgal V, Delproposto Z, Haacke EM et al (2005) Clinical applications of neuroimaging with susceptibility-weighted imaging. J Magn Reson Imagin 22:439-450

18. Zhang W, Zhao J, Guo D et al (2010) Application of susceptibility weighted imaging in revealing intratumoral blood products and grading gliomas. J Radiol 91:485-490

19. Kickingereder P, Sahm F, Radbruch A et al (2015) IDH mutation status is associated with a distinct hypoxia/angiogenesis transcriptome signature which is non-invasively predictable with rCBV imaging in human glioma. Scientific reports 5:16238

20. Chawla S, Krejza J, Vossough A et al (2013) Differentiation between oligodendroglioma genotypes using dynamic susceptibility contrast perfusion-weighted imaging and proton MR spectroscopy. Am J Neuroradiol 34:1542-1549

21. Law M, Brodsky JE, Babb J et al (2007) High cerebral blood volume in human gliomas predicts deletion of chromosome 1p: preliminary results of molecular studies in gliomas with elevated perfusion. J Magn Reson Imagin 25:1113-1119

22. Emblem KE, Nedregaard B, Nome T et al (2008) Glioma grading by using histogram analysis of blood volume heterogeneity from MR-derived cerebral blood volume maps. Radiology 247:808-817

23. Usinskiene J, Ulyte A, Bjørnerud A et al (2016) Optimal differentiation of high- and low-grade glioma and metastasis: a metaanalysis of perfusion, diffusion, and spectroscopy metrics. Neuroradiology 58:339-350

24. Spampinato MV, Smith JK, Kwock L et al (2007) Cerebral blood volume measurements and proton MR spectroscopy in grading of oligodendroglial tumors. Am J Roentgenol 188:204-212

25. Fellah S, Caudal D, Paula AMD et al (2013) Multimodal MR imaging (diffusion, perfusion, and spectroscopy): is it possible to distinguish oligodendroglial tumor grade and $1 \mathrm{p} / 19 \mathrm{q}$ codeletion in the pretherapeutic diagnosis? Am J Neuroradiol 34:1326-1333
26. Mrcsed MDJ, Frcr TSS, Joycec KA et al (2007) Apparent diffusion coefficients in oligodendroglial tumors characterized by genotype. J Magn Reson Imagin 26:1405-1412

27. Xiong J, Tan W, Wen J et al (2016) Combination of diffusion tensor imaging and conventional MRI correlates with isocitrate dehydrogenase $1 / 2$ mutations but not $1 \mathrm{p} / 19 \mathrm{q}$ genotyping in oligodendroglial tumours. Eur Radiol 26:1705-1715

28. Xu M, See SJ, Ng WH et al (2005) Comparison of magnetic resonance spectroscopy and perfusion-weighted imaging in presurgical grading of oligodendroglial tumors. Neurosurgery 56:919-926

29. Park MJ, Kim HS, Jahng GH et al (2009) Semiquantitative assessment of Intratumoral susceptibility signals using non-contrastenhanced high-field high-resolution susceptibility-weighted imaging in patients with gliomas: comparison with MR perfusion imaging. Am J Neuroradiol 30:1402-1408

30. Ding Y, Xing Z, Liu B et al (2014) Differentiation of primary central nervous system lymphoma from high-grade glioma and brain metastases using susceptibility-weighted imaging. Brain \& Behavior 4:841-849

31. Hervey-Jumper SL, Berger MS (2016) Maximizing safe resection of low- and high-grade glioma. J Neuro-Oncol 130:1-14

32. Shaw EG, Scheithauer BW, O'Fallon JR (1997) Supratentorial gliomas: a comparative study by grade and histologic type. J NeuroOncol 31:273-278

33. Nijjar TS, Simpson WJ, Gadalla T et al (1993) Oligodendroglioma. The Princess Margaret Hospital experience (1958-1984). Cancer 71:4002-4006

34. Douw L, Klein M, Fagel SS et al (2009) Cognitive and radiological effects of radiotherapy in patients with low-grade glioma: long-term follow-up. Lancet Neurol 8:810-818

35. Leighton C, Fisher B, Bauman G et al (1997) Supratentorial lowgrade glioma in adults: an analysis of prognostic factors and timing of radiation. J Clini Oncol 15:1294-1301

36. Bent MJVD (2006) Adjuvant procarbazine, lomustine, and vincristine improves progression-free survival but not overall survival in newly diagnosed anaplastic oligodendrogliomas and oligoastrocytomas: a randomized European Organisation for Research and Treatment of Cancer Ph. J Clin Oncol 24:2715-2722

37. Barker FG, Chang SM, Huhn SL et al (2015) Age and the risk of anaplasia in magnetic resonance-nonenhancing supratentorial cerebral tumors. Cancer 80:936-941

38. Sonoda Y, Shibahara I, Kawaguchi T et al (2015) Association between molecular alterations and tumor location and MRI characteristics in anaplastic gliomas. Brain Tumor Pathol 32:99-104

39. Brown R, Zlatescu M, Sijben A et al (2008) The use of magnetic resonance imaging to noninvasively detect genetic signatures in oligodendroglioma. Clin Cancer Res 14:2357-2362

40. Saito T, Muragaki Y, Maruyama T et al (2016) Calcification on CT is a simple and valuable preoperative indicator of $1 p / 19 q$ loss of heterozygosity in supratentorial brain tumors that are suspected grade II and III gliomas. Brain Tumor Pathol 33:1-8

41. Johnson DR, Diehn FE, Giannini C et al (2017) Genetically defined oligodendroglioma is characterized by indistinct tumor borders at MRI. Am J Neuroradiol. doi:10.3174/ajnr.A5070

42. Schoenegger K, Oberndorfer S, Wuschitz B et al (2009) Peritumoral edema on MRI at initial diagnosis: an independent prognostic factor for glioblastoma? Eur J Neurol 16:874-878

43. An C, Idema AJ, Wesseling P (2007) Diffuse glioma growth: a guerilla war. Acta Neuropathol 114:443-458

44. Bammer R (2003) Basic principles of diffusion-weighted imaging. Eur J Radiol 45:169-184

45. Whitmore RG, Krejza J, Kapoor GS et al (2007) Prediction of oligodendroglial tumor subtype and grade using perfusion weighted magnetic resonance imaging. J Neurosurg 107:600-609

46. Prensner JR, Chinnaiyan AM (2011) Metabolism unhinged: IDH mutations in cancer. Nat Med 17:291-293 